\title{
Dual combinatorics of zonal polynomials
}

\author{
Valentin Féray $^{1}$ and Piotr Śniady ${ }^{2,3}$ \\ ${ }^{1}$ LaBRI, cnrs, Université Bordeaux 1, Talence, France \\ ${ }^{2}$ Instytut Matematyczny, Uniwersytet Wrocławski, Wroctaw, Poland \\ ${ }^{3}$ Instytut Matematyczny, Polska Akademia Nauk, Warszawa, Poland
}

\begin{abstract}
In this paper we establish a new combinatorial formula for zonal polynomials in terms of power-sums. The proof relies on the sign-reversing involution principle. We deduce from it formulas for zonal characters, which are defined as suitably normalized coefficients in the expansion of zonal polynomials in terms of power-sum symmetric functions. These formulas are analogs of recent developments on irreducible character values of symmetric groups. The existence of such formulas could have been predicted from the work of M. Lassalle who formulated two positivity conjectures for Jack characters, which we prove in the special case of zonal polynomials.

Résumé. Dans cet article, nous établissons une nouvelle formule combinatoire pour les polynômes zonaux en fonction des fonctions puissance. La preuve utilise le principe de l'involution changeant les signes. Nous en déduisons des formules pour les caractères zonaux, qui sont définis comme les coefficients des polynômes zonaux écrits sur la base des fonctions puissance, normalisés de manière appropriée. Ces formules sont des analogues de développements récents sur les caractères du groupe symétrique. L'existence de telles formules aurait pu être prédite à partir des travaux de M. Lassalle, qui a proposé deux conjectures de positivité sur les caractères de Jack, que nous prouvons dans le cas particulier des polynômes zonaux.
\end{abstract}

Keywords: zonal polynomials, zonal characters, Jack polynomials, Kerov polynomials, Stanley polynomials

The results of this extended abstract correspond to the ones of the full paper [FŚ11b], which will be published elsewhere. However, the method of proof used here is different and new.

\section{Introduction}

Zonal polynomials were introduced by James [Jam60, Jam61] (who credits also Hua [Hua63]) in order to solve some problems from statistics and multivariate analysis. They quickly became a fundamental tool in this theory as well as in the random matrix theory (an overview can be found in the book of Muirhead [Mui82]). They also play an important role in representation theory: they appear as zonal spherical functions of the pairs $\left(\mathfrak{S}_{2 n}, H_{n}\right)$ (where $H_{n}$ is the hyperoctahedral group) and $\left(\mathrm{GL}_{d}(\mathbb{R}), O_{d}\right)$, which means that they describe a canonical basis of the algebra of left and right $H_{n}$-invariant (resp. $O_{d^{-}}$ invariant) functions on $\mathfrak{S}_{2 n}$ (resp. $\mathrm{GL}_{d}(\mathbb{R})$ ). This last property shows that zonal polynomials can be viewed as an analogue of Schur symmetric functions since the latter are zonal spherical functions for the Gelfand pairs $\left(\mathfrak{S}_{n} \times \mathfrak{S}_{n}, \mathfrak{S}_{n}\right)$ and $\left(\mathrm{GL}_{d}(\mathbb{C}), U_{d}\right)$. Besides, many of the properties of Schur functions can be extended to zonal polynomials and this article goes in this direction.

The main result of this article is a new combinatorial formula for zonal polynomials (Theorem 3 ). Note that, as the latter are a particular case of Jack symmetric functions, there already exists a combinatorial 
interpretation for them in terms of ribbon tableaux (due to Stanley [Sta89]). But our formula is of different kind: using it, one can describe combinatorially the coefficients of the zonal polynomial $Z_{\lambda}$ expanded in the power-sum basis as a function of $\lambda$. In more concrete words, the combinatorial objects describing the coefficient of $p_{\mu}$ in $Z_{\lambda}$ depend on $\mu$, whereas the statistics on them depend on $\lambda$ (in Stanley's result it is roughly the opposite). Note that the ring of shifted symmetric functions [0097] is involved in this dual approach [Las08, Proposition 2]. The situation is analogous to recent developments concerning characters of the symmetric groups.

Zonal polynomials are known to be the special case of Jack symmetric functions. We conjecture that there exists some formula for Jack polynomials extending the one we present here for zonal polynomials, but unfortunately we have not been able to find it: even if our proof uses only general properties of Jack polynomials, we really need the special value of the parameter corresponding to zonal polynomials.

The paper is organized as follows. In Section 2 we recall the necessary definitions. In Section 3 we state our main theorem and in Section 4 we present its proof. Then, in Section 5 we sketch a few consequences of this theorem on zonal characters.

\section{Notations}

In this section, we give some definitions and notations on symmetric functions in general, on Jack polynomials and, in particular, zonal polynomials. We also give a few definitions of pair-partitions, which are the combinatorial objects involved in our formulas.

\subsection{Symmetric functions}

As much as possible, we use the notations of I.G. Macdonald's book [Mac95].

By definition, a partition $\lambda=\left(\lambda_{1}, \lambda_{2}, \ldots\right)$ of the integer $n$ is a non-increasing sequence of nonnegative integers of sum $n$. Its length $\ell(\lambda)$ is the number of non-zero terms in the sequence. For $i \geq 1$ we denote by $m_{i}(\lambda)$ the number of occurrences of $i$ in $\lambda$. Finally, let us define aut $(\lambda):=\prod_{i \geq 1} m_{i}(\lambda)$ ! and $z_{\lambda}=\operatorname{aut}(\lambda) \cdot \prod_{j} \lambda_{j}$.

The ring $\Lambda$ of symmetric functions has several classical linear bases, all indexed by partitions:

- monomial symmetric functions: let us use for monomials the short notation $\mathbf{x}^{\mathbf{v}}=x_{1}^{v_{1}} x_{2}^{v_{2}} \ldots$, then

$$
m_{\lambda}:=\sum_{\mathbf{v} \in S_{\infty}(\lambda)} \mathbf{x}^{\mathbf{v}},
$$

where the sum runs over all vectors $\mathbf{v}$ which are permutations of $\lambda$ (without multiplicities);

- power-sum symmetric functions: they are defined by

$$
p_{0}=1, \quad p_{k}=\sum_{i} x_{i}^{k}, \quad p_{\mu}=\prod_{j} p_{\mu_{j}} ;
$$

- Schur functions $\left(s_{\lambda}\right)$ : they have several equivalent definitions, one of them will be given in the next paragraph.

In addition to the additive and multiplicative structures, the ring of symmetric functions can be endowed with a scalar product (called Hall scalar product), for which:

$$
\left\langle p_{\lambda}, p_{\mu}\right\rangle=z_{\lambda} \delta_{\lambda, \mu}, \quad\left\langle s_{\lambda}, s_{\mu}\right\rangle=\delta_{\lambda, \mu} .
$$




\subsection{Jack symmetric functions and zonal polynomials}

The set of partitions of a given integer $n$ can be endowed with a partial order, called dominance order: by definition,

$$
\lambda \preceq \mu \Longleftrightarrow \forall_{i \geq 1} \lambda_{1}+\cdots+\lambda_{i} \leq \mu_{1}+\cdots+\mu_{i} .
$$

With respect to this order, the matrix of Schur functions in the monomial basis is upper triangular: i.e., if one writes a Schur functions $s_{\lambda}$ on the monomial basis, the indices of the monomial symmetric functions with non-zero coefficients are dominated by $\lambda$. This triangularity property, together with the fact that they form an orthonormal basis with respect to Hall scalar product, determines entirely the Schur functions. Indeed, one can construct them by choosing any total order refining the dominance order and applying Gram-Schmidt orthogonalization process to the monomial symmetric function basis.

Hall scalar product can be deformed in the following way: $\langle\cdot, \cdot\rangle_{\alpha}$ is the bilinear form whose values on the power-sum basis is given by

$$
\left\langle p_{\lambda}, p_{\mu}\right\rangle_{\alpha}=z_{\lambda} \alpha^{\ell(\lambda)} \delta_{\lambda, \mu} .
$$

The following result corresponds to equations (VI,10.13) and (VI,10.14) of Macdonald's book [Mac95].

Proposition 1 Fix $\alpha>0$. There exists a (unique) family of functions $J_{\lambda}^{(\alpha)}$ such that the following three conditions are fulfilled:

TR The matrix of $J_{\lambda}^{(\alpha)}$ written in the monomial basis is upper triangular.

OR The $J_{\lambda}^{(\alpha)}$ form an orthogonal basis of the ring of symmetric functions endowed with the scalar product $\langle\cdot, \cdot\rangle_{\alpha}$.

$N$ The coefficient of $p_{1}^{|\lambda|}$ in the power sum expansion of $J_{\lambda}^{(\alpha)}$ is equal to 1.

These functions are called Jack symmetric functions. When $\alpha=1$ they correspond, up to multiplication by a scalar, to Schur functions. When $\alpha=2$ they are called zonal polynomials (because, as we already mentioned in Section 11, they appear in representation theory of some Gelfand pairs, see [Mac95, VII, 2]) and denoted $Z_{\lambda}$.

The uniqueness of such a family is easy to prove: it is essentially the same argument as the one given above for the characterization of Schur functions.

\subsection{Pair partitions}

Definition 2 A pair-partition of $[2 n]=\{1, \ldots, 2 n\}$ is a set of pairwise disjoint two-element sets, such that their (disjoint) union is equal to $[2 n]$.

A pair-partition $S$ can be also seen as an involution as the set $[2 n]$. Therefore, we use the notation $S(i)$ for the partner of $i$, i.e. the other element in the same set of $S$.

For two pair-partitions $S_{1}, S_{2}$ of the same set [2n] we consider the edge-bicolored graph $G_{S_{1}, S_{2}}$ defined as follows: its vertex set is $[2 n]$, its red (resp. blue) edges are the pairs in $S_{1}$ (resp. $S_{2}$ ). As every vertex has degree 2 , this graph is a disjoint union of loops. We denote $\mathcal{L}\left(S_{1}, S_{2}\right)$ the corresponding set-partition of $[2 n]$. Note that the length of each loop (or equivalently the size of each block of $\mathcal{L}\left(S_{1}, S_{2}\right)$ ) is an even number because of the edge bicoloration.

It is common to associate to a set-partition the integer partition formed by the non-increasing sequence of the part sizes. Here, as we deal only with set partitions $\Pi$ with parts of even length, we will rather 
consider the non-increasing sequence of the half-sizes of the parts. This integer partition is called the type of the set partition $\Pi$ and is denoted $\mu(\Pi)$.

In other words, the type of $\mathcal{L}\left(S_{1}, S_{2}\right)$ is the partition $\ell_{1}, \ell_{2}, \ldots$ where $\left(2 \ell_{i}\right)_{i \geq 1}$ is the non-increasing sequence of the lengths of the loops of $G_{S_{1}, S_{2}}$. We also define the sign of a couple of pair-partitions as follows:

$$
(-1)^{\left(S_{1}, S_{2}\right)}=(-1)^{\left(\ell_{1}-1\right)+\left(\ell_{2}-1\right)+\cdots}=(-1)^{n-\left|\mathcal{L}\left(S_{1}, S_{2}\right)\right|} .
$$

Finally we use the short notations $p_{\Pi}=p_{\mu(\Pi)}$ and $m_{\Pi}=m_{\mu(\Pi)}$ for symmetric functions.

Example. We consider

$$
\begin{aligned}
& S_{1}=\{\{1,2\},\{3,4\},\{5,6\}\} ; \\
& S_{2}=\{\{1,3\},\{2,4\},\{5,6\}\} .
\end{aligned}
$$
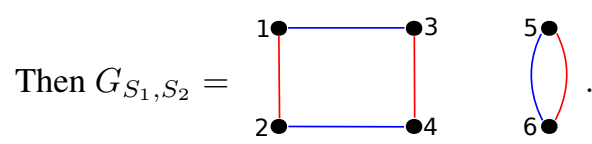

In this case, $\mathcal{L}\left(S_{1}, S_{2}\right)=\{\{1,2,3,4\},\{5,6\}\}$ and $\mu\left(\mathcal{L}\left(S_{1}, S_{2}\right)\right)=(2,1)$.

\section{The main result}

\subsection{Zonal polynomials in terms of pair-partition}

Let $\lambda=\left(\lambda_{1}, \lambda_{2}, \ldots\right)$ be a partition of $n$ and $T$ be a bijective filling of the Young diagram $2 \lambda=$ $\left(2 \lambda_{1}, 2 \lambda_{2}, \ldots\right)$ with the elements of $[2 n]$. Any pair-partition of $[2 n]$ can be viewed as a pairings of the boxes of $T$. We denote $S(T)$ the pair partition which matches the boxes $(i, 2 j-1)$ and $(i, 2 j)$ of $T$ for all values of $i$ and $1 \leq j \leq \lambda_{i}$. Besides, a couple of pair-partitions $\left(S_{1}, S_{2}\right)$ is called $T$-admissible if each pair of boxes matched by $S_{2}$ is in the same row and if $S_{1}$ matches elements of the $2 j-1$-th column of $T$ with elements of its $2 j$-th column.

Theorem 3 Let $\lambda$ be a partition and $T$ be a bijective filling of $2 \lambda$. One has:

$$
Z_{\lambda}=\sum_{\left(S_{1}, S_{2}\right)}(-1)^{\left(S(T), S_{1}\right)} p_{\mathcal{L}\left(S_{1}, S_{2}\right)} .
$$

Example. Let $\lambda=(2,1)$ and $T=$\begin{tabular}{|l|l|l|l|}
\hline 1 & 2 & 3 & 4 \\
\hline 5 & 6 &
\end{tabular} . Then $\left(S_{1}, S_{2}\right)$ is $T$-admissible if and only if:

$$
\begin{aligned}
S_{1} \in\left\{\{\{1,2\},\{3,4\},\{5,6\}\}, \quad \text { and } \quad S_{2} \in\{\right. & \{\{1,2\},\{3,4\},\{5,6\}\}, \\
\{\{1,6\},\{3,4\},\{2,5\}\}\} & \{\{1,3\},\{2,4\},\{5,6\}\}, \\
& \{\{1,4\},\{2,3\},\{5,6\}\}\} .
\end{aligned}
$$

The first possible value of $S_{1}$ gives $(-1)^{\left(S(T), S_{1}\right)}=1$ and the types of $\mathcal{L}\left(S_{1}, S_{2}\right)$ for the three possible values of $S_{2}$ are, respectively, $(1,1,1),(2,1)$ and $(2,1)$. For the second value of $S_{1}$ one has $(-1)^{\left(S(T), S_{1}\right)}=-1$ and the types of the corresponding set-partitions $\mathcal{L}\left(S_{1}, S_{2}\right)$ are, respectively, $(2,1)$, (3) and (3).

Finally, one obtains $Z_{(2,1)}=p_{(1,1,1)}+p_{(2,1)}-2 p_{(3)}$.

Remark. This theorem is an analogue of a known result on Schur symmetric functions:

$$
\frac{n ! \cdot s_{\lambda}}{\operatorname{dim}(\lambda)}=\sum(-1)^{\left|\sigma_{1}\right|} p_{\text {type }\left(\sigma_{1} \circ \sigma_{2}\right)},
$$


where the sum runs over pairs of permutations $\left(\sigma_{1}, \sigma_{2}\right)$ of the boxes of the diagram $\lambda$ such that $\sigma_{1}$ (resp. $\left.\sigma_{2}\right)$ preserves the columns (resp. the rows) of $\lambda$ and type $\left(\sigma_{1} \circ \sigma_{2}\right)$ denotes the partition describing the lengths of the cycles of $\sigma_{1} \circ \sigma_{2}$. This formula is a consequence of the explicit construction of the representation associated to $\lambda$ via the Young symmetrizer. For a detailed proof, see [FŚ11a, Theorem 4]. In [Han88], the authors tries unsuccessfully to generalize it to Jack polynomials by introducing some statistics on couples of permutations. Our result shows, that, at least for $\alpha=2$, a natural way to generalize it consists in using other combinatorial objects than permutations.

\subsection{Application}

Let us look at the coefficients $\theta_{\nu}^{(\alpha)}(\lambda)$ of the power sum expansion of Jack polynomials:

$$
J_{\lambda}^{(\alpha)}=\sum \theta_{\nu}^{(\alpha)}(\lambda) p_{\nu}
$$

In the case $\alpha=1$ we obtain, up to a multiplicative constant, the irreducible characters of the symmetric groups. By analogy, in the general case, we call these quantities Jack characters (or zonal characters in the case $\alpha=2$ ). With the right choice of the normalizing constant, namely

$$
\Sigma_{\nu}^{(\alpha)}(\lambda)=\left(\begin{array}{c}
|\lambda|-|\nu|+m_{1}(\nu) \\
m_{1}(\nu)
\end{array}\right) z_{\nu} \theta_{\nu, 1|\lambda|-|\nu|}^{(\alpha)}(\lambda),
$$

they are $\alpha$-shifted symmetric functions of the partition $\lambda$.

Recently, M. Lassalle stated two positivity conjectures [Las08, Las09] suggesting the existence of some combinatorial description of this coefficient. Theorem 3 can be used to obtain such a combinatorial description in the particular case of zonal polynomials. Note that this particular case is especially interesting because the quantities $\theta_{\nu}^{(2)}(\lambda)$ have representation-theoretical interpretations (see [Mac95, VII,2]). We give precise statements in Section 5 .

\subsection{Combinatorial interpretation}

The triplet of pair-partitions $\left(S(T), S_{1}, S_{2}\right)$ can be seen as a map (bipartite graph drawn on a - possibly non-orientable - two-dimensional, borderless surface), see [GJ96]. The statistics considered in Theorem 3. Theorem 9. Theorem 10 and Theorem 11 are natural in this context: $\mathcal{L}\left(S(T), S_{1}\right)$ and $\mathcal{L}\left(S(T), S_{2}\right)$ correspond respectively to white and black vertices, while $\mathcal{L}\left(S_{1}, S_{2}\right)$ corresponds to the faces.

Therefore, our results can be rephrased in a natural way using graphs on surfaces. For more details and precise statements see the complete version of this paper [FŚ11b].

\subsection{Links between pair-partitions and zonal characters}

It should be stressed that a previous link between pair-partitions and zonal characters can be found in the work of Goulden and Jackson [GJ96]. But their result goes in the opposite direction than ours: they count pair-partitions using zonal characters, while we express zonal characters using pair-partitions. The same picture exists for permutations and usual characters. It would be nice to understand the link between these two dual approaches. 


\section{Proof of Theorem 3}

From now on we fix $\alpha=2$. Let us define $Y_{\lambda}$ as the right-hand side of Theorem 3 (it obviously does not depend on the choice of $T$ ). In this section we prove that $Y_{\lambda}$ satisfies the three properties of Proposition 1 . In this way we prove Theorem 3 and we also give an alternative proof of Proposition 1 in the special case $\alpha=2$.

\subsection{Combinatorial lemmas}

The proof relies on three simple combinatorial lemmas.

Lemma 4 Let $\lambda$ and $\mu$ be two partitions of the same integer $n$. If $\lambda$ does not dominate $\mu$ then for any bijective fillings $T_{\lambda}$ and $T_{\mu}$ of the diagrams $\lambda$ and $\mu$ with integers in $[n]$ there exist two elements $i$ and $j$ which are in the same row of $T_{\mu}$ and in the same column of $T_{\lambda}$.

Proof: Let us replace in the tableau $T_{\lambda}$ each number $i$ by the index of the row of $T_{\mu}$ containing $i$. We have now a filling $T_{\lambda}^{\prime}$ of $\lambda$ in which any number $k$ appears $\mu_{k}$ times and we have to prove the existence of a column with some number appearing (at least) twice. Of course, reordering each column of $T_{\lambda}^{\prime}$ in the increasing order does not change anything. As $\lambda$ does not dominate $\mu$, there exists an integer $i$ such that:

$$
\mu_{1}+\cdots+\mu_{i}>\lambda_{1}+\cdots+\lambda_{i}
$$

But $\mu_{1}+\cdots+\mu_{i}$ is exactly the number of integers smaller than $i+1$ in $T_{\lambda}^{\prime}$. There is not enough room to fit all these numbers in the first $i$ rows of $\lambda$, therefore there exists a box $\square$ in the other rows of $\lambda$ which contains a number which is smaller or equal than $i$. The column containing $\square$ has therefore at least $i+1$ boxes with entries smaller or equal to $i$. The pigeon-hole principle shows that this column must contain a repeated entry.

Consider the following action of $S_{2 n}$ on the pair-partitions of [2n]: if $\sigma$ is a permutation in $S_{2 n}$ and $S$ a pair-partition of $[2 n]$, we denote by $\sigma(S)$ the pair partition such that $\{\sigma(i), \sigma(j)\}$ is a part of $\sigma(S)$ if and only if $\{i, j\}$ is a part of $S$. Of course, the graphs $G_{S_{1}, S_{2}}$ and $G_{\sigma\left(S_{1}\right), \sigma\left(S_{2}\right)}$ are isomorphic thus the corresponding set of loops have the same type. Conversely:

Lemma 5 The couples $\left(S_{1}, S_{2}\right)$ such that $\mu\left(\mathcal{L}\left(S_{1}, S_{2}\right)\right)=\nu$ form exactly one orbit under the diagonal action of the symmetric group $S_{2 n}$. Moreover, there are exactly $\frac{(2 n) !}{z_{\nu} 2^{\ell(\nu)}}$ of them.

Proof: Let us consider two couples $\left(S_{1}, S_{2}\right)$ and $\left(S_{1}^{\prime}, S_{2}^{\prime}\right)$, such that both graphs $G:=G_{S_{1}, S_{2}}$ and $G^{\prime}:=G_{S_{1}^{\prime}, S_{2}^{\prime}}$ are collections of loops of lengths $2 \nu_{1}, 2 \nu_{2} \ldots$ These two graphs are isomorphic as edgebicolored graphs. Let $\varphi$ be any isomorphism of them. As it sends vertices of $G$ on vertices of $G^{\prime}$, it can be seen as a permutation in $S_{2 n}$. As it sends red (resp. blue) edges of $G$ on red (resp. blue) edges of $G^{\prime}$, one has: $\varphi\left(S_{1}\right)=S_{1}^{\prime}$ (resp. $\varphi\left(S_{2}\right)=S_{2}^{\prime}$ ). Thus all couples of pair-partitions of type $\nu$ are in the same orbit.

We will prove now that the size of the centralizer of a fixed couple $\left(S_{1}, S_{2}\right)$ is equal to $z_{\nu} 2^{\ell(\nu)}=$ $\prod_{i} m_{i}(\nu) !(2 i)^{m_{i}(\nu)}$. Indeed, an element in the centralizer is entirely determined by the image of one given point in each loop of $\mathcal{L}\left(S_{1}, S_{2}\right)$. Moreover, the image of an element in a loop of length $2 \ell$ must be in a loop of the same length $2 \ell$ and the images of two elements in two different loops must be in two different loops. 
Lemma 6 Let $\lambda$ be a partition of $n$, let $T$ be a bijective filling of $2 \lambda$ and let $S_{1}$ be a pair-partition matching elements of the $2 j-1$-th column of $T$ with elements in its $2 j$-th column. If $\tau=(i j)$ with $i$ and $j$ in the same column of $T$, and $S=S(T)$ then

$$
(-1)^{\left(S, \tau\left(S_{1}\right)\right)}=(-1)^{\left(S, S_{1}\right)+1} .
$$

Proof: Let us first remark that $G_{S, \tau\left(S_{1}\right)}$ can be obtained from $G_{S, S_{1}}$ by replacing the blue edges $\left\{S_{1}(i), i\right\}$ and $\left\{S_{1}(j), j\right\}$ by $\left\{S_{1}(i), j\right\}$ and $\left\{S_{1}(j), i\right\}$. We distinguish two cases:

- if $i$ and $j$ are in different loops in $G_{S, S_{1}}$ then these loops are unified into one new loop in $G_{S, \tau\left(S_{1}\right)}$. Thus $\left|\mathcal{L}\left(S, \tau\left(S_{1}\right)\right)\right|=\left|\mathcal{L}\left(S, S_{1}\right)\right|-1$ and the lemma holds in this case;

- suppose $i$ and $j$ are in the same loop in $G_{S, S_{1}}$. As $i$ and $j$ are in the same column of $T$ and both $S$ and $S_{1}$ match elements of the $2 j-1$-th column of $T$ with elements of its $2 j$-th column, the distance between $i$ and $j$ is even, where the distance is regarded as the number of steps within one loop. It is easy to check in this case that the loop containing $i$ and $j$ in $G_{S, S_{1}}$ is split into two in $G_{S, \tau\left(S_{1}\right)}$. Therefore, $\left|\mathcal{L}\left(S, \tau\left(S_{1}\right)\right)\right|=\left|\mathcal{L}\left(S, S_{1}\right)\right|+1$ and the lemma also holds in this case.

\subsection{Triangularity}

In this paragraph we prove that the functions $Y_{\lambda}$ satisfy property $\mathbf{T R}$.

Let us fix a bijective filling $T$ of $2 \lambda$ and set $S=S(T)$. The first step of the proof consist of writing

$$
Y_{\lambda}=\sum_{\left(S_{1}, S_{2}\right)}(-1)^{\left(S, S_{1}\right)} p_{\mathcal{L}\left(S_{1}, S_{2}\right)}
$$

in the monomial basis. In order to do that we will write each $p_{\mathcal{L}\left(S_{1}, S_{2}\right)}$ on this basis.

Recall that, if $\Pi$ and $\Pi^{\prime}$ are two set partitions, we say that $\Pi$ is finer than $\Pi^{\prime}$ (and write $\Pi \leq \Pi^{\prime}$ ) if each part of $\Pi^{\prime}$ is the union of some parts of $\Pi$.

Lemma 7 Let $S_{1}, S_{2}$ be two pair partitions of $\{1, \ldots, 2 n\}$. Then

$$
p_{\mathcal{L}\left(S_{1}, S_{2}\right)}=\sum_{\Pi \geq \mathcal{L}\left(S_{1}, S_{2}\right)} \text { aut }(\mu(\Pi)) m_{\Pi} .
$$

Proof: It is a consequence of [Mac95, Chapter I, equation (6.9)].

Using this lemma, one can write $Y_{\lambda}$ in the monomial basis:

$$
\begin{aligned}
Y_{\lambda} & =\sum_{\left(S_{1}, S_{2}\right)} \sum_{T \text {-admissible }} \sum_{\Pi \geq \mathcal{L}\left(S_{1}, S_{2}\right)}(-1)^{\left(S, S_{1}\right)} \text { aut }(\mu(\Pi)) m_{\Pi} \\
& =\sum_{\substack{\text { Mset. } \\
\text { partition of }[2 n]}}\left[\sum_{\substack{\left(S_{1}, S_{2}\right) \\
\mathcal{L}\left(S_{1}, S_{2}\right) \leq \Pi}}(-1)^{\left(S, S_{1}\right)}\right] \text { aut }(\mu(\Pi)) m_{\Pi} .
\end{aligned}
$$

Remark. The inequality $\mathcal{L}\left(S_{1}, S_{2}\right) \leq \Pi$ is equivalent to the following local condition: the partners of any integer $i$ in the pair-partitions $S_{1}$ and $S_{2}$ are in the same part of $\Pi$ as $i$.

Let us assume now that $\mu(\Pi)$ is not dominated by $\lambda$. By Lemma 4 , there exist $i$ and $j$ in $[2 n]$, which are in the same part of $\Pi$ and in the same column of $T$. Let us denote $\tau=(i j) \in S_{2 n}$. Then: 
- $\left(\tau\left(S_{1}\right), S_{2}\right)$ is $T$-admissible $\Longleftrightarrow\left(S_{1}, S_{2}\right)$ is $T$-admissible;

- $\mathcal{L}\left(\tau\left(S_{1}\right), S_{2}\right) \leq \Pi \Longleftrightarrow \mathcal{L}\left(S_{1}, S_{2}\right) \leq \Pi$ (because of the remark above);

- $(-1)^{\left(S, \tau\left(S_{1}\right)\right)}=(-1)^{\left(S, S_{1}\right)+1}$ (see Lemma6).

Thus, $\left(S_{1}, S_{2}\right) \mapsto\left(\tau\left(S_{1}\right), S_{2}\right)$ is a sign reversing involution proving that the expression in the bracket in the right-hand side of (3) is equal to zero as soon as $\mu(\Pi)$ is not dominated by $\lambda$.

This ends the proof of property $\mathbf{T R}$.

\subsection{Orthogonality}

In this section we prove that the functions $Y_{\lambda}$ satisfy property $\mathbf{O R}$.

As the definition of $Y_{\lambda}$ does not depend on the choice of the bijective filling $T \in F(\lambda)(F(\lambda)$ is by definition the set of bijective fillings of $2 \lambda$ ), one can write:

$$
\begin{aligned}
(2 n) ! Y_{\lambda} & =\sum_{T \in F(\lambda)} \sum_{\substack{\left(S_{1}, S_{2}\right) \\
T \text {-admissible }}}(-1)^{\left(S(T), S_{1}\right)} p_{\mathcal{L}\left(S_{1}, S_{2}\right)} \\
& =\sum_{\substack{S_{1}, S_{2} \\
\text { pair-partitions }}} \sum_{\substack{T \in F(\lambda) \text { s.t. } \\
\left(S_{1}, S_{2}\right)}}(-1)^{\left(S(T), S_{1}\right)} p_{\mathcal{L}\left(S_{1}, S_{2}\right) .} .
\end{aligned}
$$

Let $\nu$ be a partition of $n$. A consequence of Lemma 5 is that each of the $\frac{(2 n) !}{z_{\nu} 2^{\ell(\nu)}}$ couples of pair-partitions $\left(S_{1}, S_{2}\right)$ such that $\mu\left(\mathcal{L}\left(S_{1}, S_{2}\right)\right)=\nu$ has the same contribution to the right-hand side of the previous equation. Therefore, if we fix such a couple $\left(S_{1}, S_{2}\right)$, the coefficient of $p_{\nu}$ in $Y_{\lambda}$ is given by:

$$
(2 n) !\left[p_{\nu}\right] Y_{\lambda}=\frac{(2 n) !}{z_{\nu} 2^{\ell(\nu)}} \sum_{\substack{T \in F(\lambda) \text { s.t. } \\\left(S_{1}, S_{2}\right)}}(-1)^{\left(S(T), S_{1}\right)} .
$$

But, using equation (4) for $Y_{\lambda}$, one has the following expression for the scalar product:

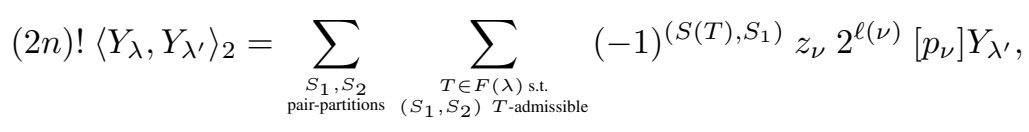

where $\nu=\mu\left(\mathcal{L}\left(S_{1}, S_{2}\right)\right)$ depends on the summation index. Now, $\left[p_{\nu}\right] Y_{\lambda^{\prime}}$ can be evaluated via equation (5) and we obtain:

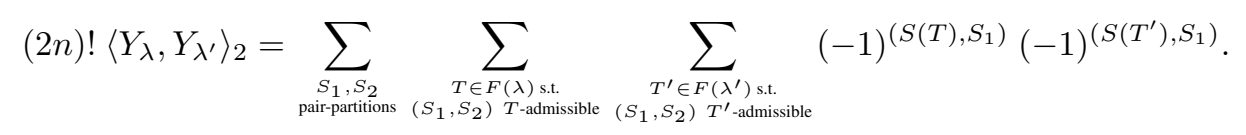

Let us assume $\lambda \neq \lambda^{\prime}$. One can assume without loss of generality that $\lambda$ is not dominated by $\lambda^{\prime}$; otherwise we swap $\lambda$ and $\lambda^{\prime}$. By Lemma 4 there exist two elements $i$ and $j$ which are in the same row of $T^{\prime}$ and in the same column of $T$. We choose the smallest ones in the lexicographic order. We denote by $\tau_{T, T^{\prime}}$ the transposition $(i j)$ and we consider the function:

$$
\varphi:\left(S_{1}, S_{2}, T, T^{\prime}\right) \mapsto\left(\tau_{T, T^{\prime}}\left(S_{1}\right), S_{2}, T, T^{\prime} \circ \tau_{T, T^{\prime}}\right),
$$

where $T^{\prime} \circ \tau_{T, T^{\prime}}$ is the tableau $T^{\prime}$ with the locations of $i$ and $j$ interchanged. It is easy to check that: 
- $\varphi$ is an involution (because $T^{\prime} \circ \tau_{T, T^{\prime}}$ and $T^{\prime}$ have the same rows and, therefore, the unordered pair $\{i, j\}$ is the same for the two pairs of fillings);

- $\left(S_{1}, S_{2}\right)$ is $T$-admissible if and only if $\left(\tau_{T, T^{\prime}}\left(S_{1}\right), S_{2}\right)$ is $T$-admissible;

- $\left(S_{1}, S_{2}\right)$ is $T^{\prime}$-admissible if and only if $\left(\tau_{T, T^{\prime}}\left(S_{1}\right), S_{2}\right)$ is $T^{\prime} \circ \tau_{T, T^{\prime}}$-admissible;

- $(-1)^{\left(S(T), \tau_{T, T^{\prime}}\left(S_{1}\right)\right)}=(-1)^{\left(S(T), S_{1}\right)+1}$ (see Lemma 6 ;

- $(-1)^{\left(S\left(T^{\prime} \circ \tau_{T, T^{\prime}}\right), \tau_{T, T^{\prime}}\left(S_{1}\right)\right)}=(-1)^{\left(S\left(T^{\prime}\right), S_{1}\right)}$ (because $\left.S\left(T^{\prime} \circ \tau_{T, T^{\prime}}\right)=\tau_{T, T^{\prime}}\left(S\left(T^{\prime}\right)\right)\right)$.

The contributions of the quadruplets $\left(S_{1}, S_{2}, T, T^{\prime}\right)$ and $\varphi\left(S_{1}, S_{2}, T, T^{\prime}\right)$ in equation (6) cancel, which proves that the functions $Y_{\lambda}$ are orthogonal with respect to the deformed Hall scalar product $\langle\cdot, \cdot\rangle_{2}$.

\subsection{Normalization}

In this paragraph we prove that the functions $Y_{\lambda}$ satisfy property $\mathbf{N}$.

Let $\lambda$ be a partition of $n$. We fix a bijective filling $T$ of $2 \lambda$. We have to find the $T$-admissible couples $\left(S_{1}, S_{2}\right)$ with $\mu\left(\mathcal{L}\left(S_{1}, S_{2}\right)\right)=1^{n}$. The last condition is equivalent to $S_{1}=S_{2}$ and a couple $\left(S_{1}, S_{1}\right)$ is $T$-admissible if and only if $S_{1}=S(T)$. In this case, $(-1)^{\left(S(T), S_{1}\right)}=1$. Therefore, $\left[p_{1^{n}}\right] Y_{\lambda}=1$ and Theorem 3 is proved.

\section{Combinatorial formulas for zonal characters}

In this section we give new formulas for zonal characters (the latter are defined in Section 3.2, which are analogues of some recent formulas for characters of the symmetric groups and establish some particular cases of some conjectures of Lassalle. These formulas can be derived relatively easily from our main result, as done in the complete version of this paper [FŚ11b].

\subsection{Via numbers of embeddings}

Let $S_{0}, S_{1}, S_{2}$ be three pair-partitions of the set $[2 k]$. We consider the following function on the set of Young diagrams:

Definition $8 N_{S_{0}, S_{1}, S_{2}}^{(1)}(\lambda)$ is the number of functions $f$ from $[2 k]$ to the boxes of the Young diagram $\lambda$ such that for every $l \in[2 k]$ :

(QO) $f(l)=f\left(S_{0}(l)\right)$, in other words $f$ can be viewed as a function on the set of pairs constituting $S_{0}$;

(Q1) $f(l)$ and $f\left(S_{1}(l)\right)$ are in the same column;

(Q2) $f(l)$ and $f\left(S_{2}(l)\right)$ are in the same row.

Theorem 9 Let $\nu$ be a partition of the integer $k$ and $S_{1}, S_{2}$ be two fixed pair-partitions of the set $[2 k]$ such that $\mu\left(\mathcal{L}\left(S_{1}, S_{2}\right)\right)=\nu$. Then one has the following equality between functions on the set of Young diagrams:

$$
\Sigma_{\nu}^{(2)}=\frac{1}{2^{\ell(\nu)}} \sum_{S_{0}}(-1)^{\left(S_{0}, S_{1}\right)} 2^{\left|\mathcal{L}\left(S_{0}, S_{1}\right)\right|} N_{S_{0}, S_{1}, S_{2}}^{(1)},
$$

where the sum runs over pair-partitions of $[2 k]$. 


\subsection{In terms of Stanley's coordinates}

The notion of Stanley's coordinates was introduced by Stanley [Sta04] who found a nice formula for normalized irreducible character values of the symmetric group corresponding to rectangular Young diagrams. In order to generalize this result, he defined, given two sequences $\mathbf{p}$ and $\mathbf{q}$ of positive integers of same size ( $q$ being non-increasing), the partition:

$$
\mathbf{p} \times \mathbf{q}=(\underbrace{q_{1}, \ldots, q_{1}}_{p_{1} \text { times }}, \ldots, \underbrace{q_{l}, \ldots, q_{l}}_{p_{l} \text { times }}) .
$$

Then he suggested to consider the quantity $\Sigma_{\nu}^{(1)}(\mathbf{p} \times \mathbf{q})$ as a polynomial in $\mathbf{p}$ and $\mathbf{q}$. An explicit combinatorial interpretation of the coefficients was conjectured in [Sta06] and proved in [Fér10].

It is easy to deduce from the above expansion of $\Sigma_{\nu}^{(2)}$ in terms of the $N$ functions a combinatorial description of the polynomial $\Sigma_{\nu}^{(2)}(\mathbf{p} \times \mathbf{q})$.

Theorem 10 Let $\nu$ be a partition of the integer $k$ and $S_{1}, S_{2}$ be two fixed pair-partitions of $[2 k]$ such that $\mu\left(\mathcal{L}\left(S_{1}, S_{2}\right)\right)=\nu$. Then, one has:

$$
\Sigma_{\nu}^{(2)}(\mathbf{p} \times \mathbf{q})=\frac{(-1)^{k}}{2^{\ell(\nu)}} \sum_{S_{0}}\left[\sum_{\phi: \mathcal{L}\left(S_{1}, S_{0}\right) \rightarrow \mathbb{N}^{*}} \prod_{l \in \mathcal{L}\left(S_{1}, S_{0}\right)}\left(p_{\varphi(l)}\right) \cdot \prod_{l^{\prime} \in \mathcal{L}\left(S_{2}, S_{0}\right)}\left(-2 q_{\psi\left(l^{\prime}\right)}\right)\right]
$$

where $\psi\left(l^{\prime}\right):=\max _{l} \varphi(w)$ with l running over the loops of $\mathcal{L}\left(S_{0}, S_{1}\right)$ having at least one element in common with $l^{\prime}$.

\subsection{In terms of free cumulants}

The following observation is due to Lassalle [Las09]. Let $k \geq 1$ be a fixed integer and let $\alpha$ be fixed. Since $\Sigma_{k}^{(\alpha)}$ is an $\alpha$-shifted symmetric function and the anisotropic free cumulants $\left(R_{l}^{(\alpha)}\right)_{l \geq 2}$ form an algebraic basis of the ring of $\alpha$-shifted symmetric functions (see [Las09] for their definition), there exists a polynomial $K_{k}^{(\alpha)}$ such that, for any Young diagram $\lambda$,

$$
\Sigma_{k}^{(\alpha)}(\lambda)=K_{k}^{(\alpha)}\left(R_{2}^{(\alpha)}(\lambda), R_{3}^{(\alpha)}(\lambda), \ldots\right) .
$$

This polynomial is called Jack Kerov polynomial.

Thus Jack Kerov polynomials express Jack characters on cycles in terms of free cumulants. For more complicated conjugacy classes it turns out to be more convenient to express not directly the characters $\Sigma_{\left(k_{1}, \ldots, k_{\ell}\right)}^{(\alpha)}$ but rather cumulant

$$
(-1)^{\ell-1} \kappa^{\mathrm{id}}\left(\Sigma_{k_{1}}^{(\alpha)}, \ldots, \Sigma_{k_{\ell}}^{(\alpha)}\right) .
$$

This gives rise to generalized Jack Kerov polynomials $K_{\left(k_{1}, \ldots, k_{\ell}\right)}^{(\alpha)}$. In the classical context $\alpha=1$ these quantities have been introduced by one of us and Rattan [RŚ08]; in the Jack case they have been studied by Lassalle [Las09]. We skip the definitions and refer to the above papers for details since generalized Kerov polynomials are not of central interest for this paper.

Using the technology developed in [DFŚ10], one can deduce from Theorem 9 a combinatorial interpretation for the coefficients of generalized zonal Kerov polynomials (i.e. generalized Jack Kerov polynomials in the case $\alpha=2$ ): 
Theorem 11 Let $\nu$ be a partition of an integer $k$ and $S_{1}, S_{2}$ be two fixed pair-partitions of $[2 k]$ such that $\mu\left(\mathcal{L}\left(S_{1}, S_{2}\right)\right)=\nu$. Consider also a sequence $s_{2}, s_{3}, \ldots$ of non-negative integers with only finitely many non-zero elements. The rescaled coefficient

$$
(-2)^{\ell(\nu)}(-1)^{|\nu|+2 s_{2}+3 s_{3}+\cdots}\left[\left(R_{2}^{(2)}\right)^{s_{2}}\left(R_{3}^{(2)}\right)^{s_{3}} \cdots\right] K_{\nu}^{(2)}
$$

of the (generalized) zonal Kerov polynomial is equal to the number of couples $\left(S_{0}, q\right)$ with the following properties:

(a) the graph of vertex set $[2 n]$ obtained by drawing an edge between $i$ and $j$ if they are in the same part of $S_{0}, S_{1}$ or $S_{2}$ is connected;

(b) the number of loops in $G_{S_{0}, S_{1}}$ is equal to $s_{2}+s_{3}+\cdots$;

(c) the number of loops in $G_{S_{0}, S_{2}}$ is equal to $s_{2}+2 s_{3}+3 s_{4}+\cdots$;

(d) $q$ is a function from $\mathcal{L}\left(S_{0}, S_{1}\right)$ to the set $\{2,3, \ldots\}$; we require that each number $i \in\{2,3, \ldots\}$ is used exactly $s_{i}$ times;

(e) for every subset $A \subset \mathcal{L}\left(S_{0}, S_{1}\right)$ of loops which is nontrivial (i.e., $A \neq \emptyset$ and $A \neq \mathcal{L}\left(S_{0}, S_{1}\right)$ ), there are more than $\sum_{v \in A}(q(v)-1)$ loops in $\mathcal{L}\left(S_{0}, S_{2}\right)$ which have a non-empty intersection with at least one loop from $A$.

\section{Conclusion}

Our result on zonal polynomials and zonal characters are analogs of recent developments on Schur polynomials and characters of symmetric groups. Lassalle's work suggests that this kind of results hold for generic values of the parameter $\alpha$, but we have not been able (yet) to generalize our argument.

Note that, unlike the proof in the full version of the paper [FŚ11b], which relies on the representation theoretical interpretation of zonal polynomials, the one presented here only uses the fact that zonal polynomials are special cases of Jack polynomials. So, if we manage to guess the good combinatorial objects for other values of $\alpha$, a similar proof technique could be used.

Another approach to the general case is proposed in [DŚ11]: M. Dołęga and the second author give a combinatorial condition for an expression of the kind of Therorem 9 to be an $\alpha$-shifted symmetric function. As $\Sigma_{\nu}^{(\alpha)}(\lambda)$ belongs to this class, this could help guessing a generalization of Therorem 9

\section{Acknowledgements}

Research of PŚ was supported by the Polish Ministry of Higher Education research grant N N201 364436 for the years 2009-2012.

\section{References}

[DFŚ10] M. Dołęga, V. Féray, and P. Śniady. Explicit combinatorial interpretation of Kerov character polynomials as numbers of permutation factorizations. Adv. Math., 225(1):81-120, 2010.

[DŚ11] Maciej Dołęga and Piotr Śniady. Polynomial functions on young diagrams arising from bipartite graphs. DMTCS proceddings, FPSAC(23), 2011. 
[Fér10] V. Féray. Stanley's formula for characters of the symmetric group. Annals of Combinatorics, 13(4):453-461, 2010.

[FŚ11a] Valentin Féray and Piotr Śniady. Asymptotics of characters of symmetric groups related to Stanley character formula. Ann. Math., 173(2):887-906, 2011.

[FŚ11b] Valentin Feray and Piotr Śniady. Zonal polynomials via Stanley-Féray formula and free cumulants. Jour. Algebra, 334:338-373, 2011.

[GJ96] I. P. Goulden and D. M. Jackson. Maps in locally orientable surfaces, the double coset algebra, and zonal polynomials. Canad. J. Math., 48(3):569-584, 1996.

[Han88] Phil Hanlon. Jack symmetric functions and some combinatorial properties of Young symmetrizers. J. Combin. Theory Ser. A, 47(1):37-70, 1988.

[Hua63] L. K. Hua. Harmonic analysis of functions of several complex variables in the classical domains. Translated from the Russian by Leo Ebner and Adam Korányi. American Mathematical Society, Providence, R.I., 1963.

[Jam60] Alan T. James. The distribution of the latent roots of the covariance matrix. Ann. Math. Statist., 31:151-158, 1960.

[Jam61] Alan T. James. Zonal polynomials of the real positive definite symmetric matrices. Ann. of Math. (2), 74:456-469, 1961.

[Las08] M. Lassalle. A positivity conjecture for Jack polynomials. Math. Res. Let., 15(4):661-681, 2008.

[Las09] M. Lassalle. Jack polynomials and free cumulants. Adv. Math., 222(6):2227-2269, 2009.

[Mac95] I. G. Macdonald. Symmetric functions and Hall polynomials. Oxford Mathematical Monographs. The Clarendon Press Oxford University Press, New York, second edition, 1995. With contributions by A. Zelevinsky, Oxford Science Publications.

[Mui82] Robb J. Muirhead. Aspects of multivariate statistical theory. John Wiley \& Sons Inc., New York, 1982. Wiley Series in Probability and Mathematical Statistics.

[0O97] A. Okounkov and G. Olshanski. Shifted Jack polynomials, binomial formula, and applications. Math. Res. Lett., 4(1):69-78, 1997.

[RŚ08] A. Rattan and P. Śniady. Upper bound on the characters of the symmetric groups for balanced Young diagrams and a generalized Frobenius formula. Adv. Math., 218(3):673-695, 2008.

[Sta04] Richard P. Stanley. Irreducible symmetric group characters of rectangular shape. Sém. Lothar. Combin., 50:Art. B50d, 11 pp. (electronic), 2003/04.

[Sta89] Richard P. Stanley. Some combinatorial properties of Jack symmetric functions. Adv. Math., 77(1):76-115, 1989.

[Sta06] Richard P. Stanley. A conjectured combinatorial interpretation of the normalized irreducible character values of the symmetric group. Preprint arXiv:math.CO/0606467, 2006. 